\title{
Biosynthesis, Characterization and Antagonistic Applications of Extracellular Melanin Pigment from Marine Nocardiopsis Sps
}

\author{
Neethu Kamarudheen, Thahiya Naushad, Kokati Venkata Bhaskara Rao* \\ Department of Biomedical Sciences, School of Biosciences and Technology, VIT University, Vellore, Tamil Nadu, INDIA.
}

\begin{abstract}
Context: Although there is an abundance of several microbial phyla in the oceans actinobacteria have emerged as a major source for natural products. Streptomyces sps is the widely encountered actinobacteria. Lately, Streptomyces are increasingly been used for pigment extraction. Aim: The aim herein was to extract bioactive melanin pigment from rare actinobacteria, which is a not a widespread occurrence. Materials and Methods: Marine samples were collected aseptically from the waters of the Arabian Sea, Allepey, Kerala, India. Isolation was performed by serial dilution method upon dry heat treatment and pre-enrichment technique on Actinomycetes Isolation Agar. Selected potent isolates were fermented and the pigments were extracted. Thereafter the pigment was analyzed by various techniques viz., thin layer chromatography, UV-Visible spectrophotometry and Fourier Transfer Infra Red spectroscopy. Further, the pigment was evaluated for its antibacterial, anti-biofilm and anti-quorum sensing potential. Results: The isolates were designated as JN1 and JN2 and their respective extracts JN1M and JN2M. JN1M was found to be inhibiting biofilm forming clinical isolate Staphylococcus sp. showing an activity of $64.20 \pm 3.33 \%$ and pigment from JN2M $65.99 \pm 2.81 \%$. The melanin pigment also exhibited considerable activity against various human pathogens. The isolate JN1 was identified as Nocardiopsis dassonvillei strain JN1 (accession number: KX263302) and JN2 as Nocardiopsis sp (accession number: KX263303) by conventional and molecular techniques. Conclusion: With reference to previous reports, this is one of the very few reports of marine Nocardiopsis dassonvillei exhibiting melanin production. Thus it has established the production of melanin from rare actinomycetes, $N$. dassonvillei in specific.
\end{abstract}

Key words: Melanin, Nocardiopsis dassonvillei, Anti-quorum sensing, Anti-biofilm, FTIR.

Key Messages: There has been a step up in the demand for microbial pigments. Melanin is increasingly being used in biomedicine (against cancer, Parkinson's disease, as antimicrobials) and in cosmetic industries. We herein investigated the production of melanin from rare actinobacteria which is an uncommon occurrence. The ability of the pigment to determine anti-quorum sensing activity is a research highlight.

\section{INTRODUCTION}

The marine realm hosts diverse life forms including plants, animals and microorganisms. Among the marine microorganisms, actinobacteria are well documented for the production of chemically diverse bioactive compounds. ${ }^{1-2}$ Marine actinobacteria Dietzia, Marinispora, Nocardiopsis, Rhodococcus, Salinispora, Streptomycetes, etc. are being increasingly exploited for the discovery of structurally and functionally different bioactive substances. ${ }^{3}$ Among the microbial bioactive compounds that have been obtained so far more than 10000 bioactive compounds (enzymes, enzyme inhibitors, antibiotics, pigments and single cell proteins) are reported from actinobacteria and they are noteworthy as structurally diverse secondary metabolite producers. ${ }^{4-5}$
Submission Date: 05-04-2018; Revision Date: 14-08-2018; Accepted Date: 23-10-2018

DOI: 10.5530/ijper.53.2s.55 Correspondence:

Dr. KV Bhaskara Rao, Professor, Department of Biomedical Sciences, School of Biosciences and Technology, VIT University, Vellore, Tamil Nadu, INDIA. Phone: +91-9894350824 E-mail: kvbhaskararao@ vit.ac.in

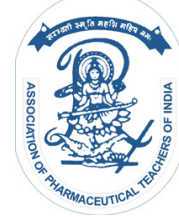

www.ijper.org 
Bacterial pigment production is one of the emerging fields of research. Actinobacteria showed considerable interest owing to their ability to produce pigments. Microbial pigment production by fermentation technology is more dynamic and economic. These pigments are biodegradable, less toxic and highly stable than synthetic and plant pigments. Microbial pigments have wide industrial application as food colourants, dying agents and as pharmaceutical agents. ${ }^{6}$ Pigments like carotenoids, anthraquinone, zeaxanthin, lycopene, melanin etc. have been synthesized from microorganisms. ${ }^{7}$

Actinobacteria are known for producing dark coloured pigments melanin or melanoid which is a benchmark for taxonomical characterization. ${ }^{8}$ Biosynthesis of melanin in lower organisms is by tyrosinase concept. In the presence of enzyme tyrosinase and oxygen, tyrosine gets oxidized to 3, 4-dihydroxyphenylalanine (DOPA) and is converted to DOPA-quinone by the further oxidation process. After undergoing several reactions DOPAquinone is converted to an indole derivative which polymerizes to form melanin. ${ }^{9-11}$

Recent studies revealed that the function of melanin is associated with the protection against environmental stress. Melanin-producing bacteria are found to be more resistant to antibiotics. These properties make melanin an important bioactive material with various industrial applications. Studies also revealed the antibacterial and antiviral properties of melanin which opens a new area in the field of research. Melanin and its derivatives are used for the therapeutic agents to cure neurodegenerative diseases like Alzheimer's disease, retinitis pigmentosa, schizophrenia and dementia. ${ }^{12}$

Thus, in our study, we intend to explore the production of melanin, a biotechnologically relevant biomolecule. Although Streptomyces spp are widespread producers of melanin, the contribution of rare actinobacteria in this is comparatively less. This makes us explore the potential of rare actinobacteria in melanin production. Nocardiopsis is a genus of rare actinobacteria that thrive in alkaline soil with high salinity. ${ }^{13}$ It has been reported to produce anti-cancer, anti-fungal, anti-candidal and anti-microbial agents. It is also interesting to note the production of tetrodotoxin. ${ }^{14-17}$ However herein the ability of production of melanin by Nocardiopsis spp is explored. The study undertaken was focused on the isolation and characterization of melanin-producing actinobacteria from marine soil sediments. It also dealt with the physical and chemical characterization of derived melanin. This is the first report on the production of melanin from marine Nocardiopsis dassonvillei.

\section{MATERIALS AND METHODS}

\section{Materials}

Dimethylsulfoxide, ferric chloride, ethyl acetate, Ninhydrin solution, absolute ethanol, methanol, disodium phosphate, ammonium molybdate, trichloroacetic acid were purchased from Sisco Research Laboratories Pvt. Ltd. (SRL) - India. Yeast extract iron agar (ISP6) and tyrosine agar base (ISP7), Starch casein agar (SCA), Actinomycetes isolation agar (AIA) were obtained from HiMedia Laboratories Pvt. Ltd., India. All the chemicals purchased were of analytical grade.

\section{Instruments}

Major instruments used for this study include UV-Visible double beam spectrophotometer 2202 (Systronics), Attenuated Total Reflectance- Fourier Transform Infra-Red spectroscopy (ATR-FTIR, Schimadzu Affnity1), Scanning Electron Microscope (SEM- Zeiss [EVO18]) and MJ Research Peltier Thermocycler.

\section{Sample collection and processing}

For isolation of actinobacteria, 2 different marine soil sediment samples were collected from the Allepey Beach, Alappuzha, $\left(9.54^{\circ} \mathrm{N}, 76.40^{\circ} \mathrm{E}\right)$. The samples were brought to the laboratory in sterile polyethene bags and were stored for further analysis at $4^{\circ} \mathrm{C}$.

\section{Isolation of rare Actinobacteria}

Three different methods were performed for the selective isolation of actinobacteria from collected marine soil samples. One gram of each sample was diluted with $9 \mathrm{~mL}$ of sterilized marine water up to $10^{-7}$ dilution. For eliminating the contamination of unwanted bacteria, Streptomyces and fungi, the dry heat treatment method was employed. The samples were heated at $120^{\circ} \mathrm{C}$ for $60 \mathrm{~min}$ in a hot air oven and 10 fold dilutions were prepared by serial dilution technique. $0.1 \mathrm{~mL}$ of the $10^{-5}$, $10^{-6}$ and $10^{-7}$ dilutions were plated on starch casein agar, actinomycetes isolation agar, tyrosine agar base (ISP7), M1 media (starch-10g, yeast extract-4g, peptone-4g, agar-18g per 1 litre of seawater) and M6 media (beef extract- $4 \mathrm{~g}$, peptone- $4 \mathrm{~g}$, yeast extract- $1 \mathrm{~g}$, glucose- $10 \mathrm{~g}$, sodium chloride- $20 \mathrm{~g}$, agar- $20 \mathrm{~g}$ per 1 litre of distilled water). In the pre-enrichment method, sterilized actinomycetes isolation broth with 1 gram of the sample was incubated at room temperature for 7 days in shaking condition at $120 \mathrm{rpm} .0 .1 \mathrm{~mL}$ of enriched broth was then inoculated onto starch casein agar, actinomycetes isolation agar, ISP7, M1 and M6 media. All the plates were observed for any growth for a period of 21 days. 


\section{Screening for melanin producing actinobacteria}

To determine the melanin production, Peptone Yeast Extract Iron agar (ISP6) and tyrosine agar base (ISP7) media were employed according to the standard protocol. All the actinobacterial isolates were streaked on ISP6 and ISP7 media for the screening of melanin-producing actinomycetes. These plates were incubated for 7 to 14 days at room temperature to observe the black diffusible pigment in media. From 20 distinct isolates, one potential melanin producer was selected for further analysis. The isolates were cultured in SS media (Soluble starch$25 \mathrm{~g}$, glucose- $10 \mathrm{~g}$, yeast extract- $2 \mathrm{~g}$, calcium carbonate- $3 \mathrm{~g}$ and trace elements- $1 \mathrm{~mL}$ ) and incubated in both shaking and static conditions for 7 to 14 days at room temperature with respective controls for observation of any extracellular pigment production.

\section{Melanin formation test and fermentation}

The standard protocol for the brown melanin formation test was followed with slight modifications. The culture broth medium was centrifuged at $8000 \mathrm{rpm}$ for $15 \mathrm{~min}$ to obtain a cell-free suspension of crude pigment. $2 \mathrm{~mL}$ of this cell-free supernatant was treated with $1 \mathrm{~mL}$ of 0.4\% L-3 4-dihydroxyphenylalanine (L-DOPA) and incubated at $37^{\circ} \mathrm{C}$ for 60 to $120 \mathrm{~min}$ to observe black colouration due to dopachrome formation. The absorbance was read spectrophotometrically at $480 \mathrm{~nm} .{ }^{18}$

Thereafter the isolates were cultured in SS broth and incubated at room temperature for 14 days at $250 \mathrm{rpm}$ for the production of crude melanin.

\section{Optimization of culture conditions for higher melanin production}

\section{Effect of carbon and nitrogen sources on melanin production}

Effect of carbon sources on melanin production was studied using five different sugars (lactose, sucrose, fructose, mannitol and galactose). For determination of the effect of nitrogen sources on melanin production, nitrogen sources such peptone, casein, L-tyrosine and L- asparagine were used. These nutrient sources were prepared in 10\% solution and were sterilized using bacteriological membrane filter. The sterilized solutions were then added to SS broth to give a final concentration of $1 \%$ of $\mathrm{C}$ and $0.1 \%$ for the $\mathrm{N}_{2}$ source. The inoculated broth cultures were incubated in a rotary shaker for 14 days at room temperature. Estimation of sugar was done with culture filtrate and L-DOPA. The dopachrome formation was read at $480 \mathrm{~nm}$ using UV-Vis spectrophotometer.

\section{Effect of temperatures on melanin production}

Melanin production by the two isolates under different temperatures was studied by incubating the inoculated cultures at $25^{\circ} \mathrm{C}, 30^{\circ} \mathrm{C}, 35^{\circ} \mathrm{C}$ and $40^{\circ} \mathrm{C}$ for 14 days. The cell-free supernatant was used for the dopachrome formation test.

\section{Effect of $\mathrm{pH}$ on melanin production}

The $\mathrm{pH}$ of SS broth was adjusted to acidic (6.0 and 6.5) neutral (7.0) and alkaline conditions (7.5, 8.0 and 8.5) and the flasks were inoculated with the two isolates to observe the effect of $\mathrm{pH}$ on melanin production.

\section{Effect of different concentrations of marine water on melanin production}

The concentrations of marine water in media were varied accordingly to $25 \%, 50 \%, 75 \%$ and $100 \%$ and its effect on melanin productions were observed with 14 days old cultural filtrate.

\section{Selective extraction and purification of melanin}

For extraction and purification of melanin, the production broth was centrifuged at $8000 \mathrm{rpm}$ for $20 \mathrm{~min}$. The cellfree supernatant was treated with $6 \mathrm{M} \mathrm{HCl}$ to precipitate the pigment at a $\mathrm{pH}$ of 2.0. After letting stand for $4 \mathrm{~h}$ the supernatant was centrifuged at $9000 \mathrm{rpm}$ for $15 \mathrm{~min}$. The precipitate obtained was washed three to four times with distilled water and centrifuged further at the same conditions. ${ }^{19}$

\section{Physiochemical characterization of melanin}

The solubility of melanin was observed by dissolving melanin $(5 \mathrm{mg} / \mathrm{mL})$ in different organic and inorganic solutions with varying polarity. Melanin dissolved in DMSO was subjected to various temperatures at $20^{\circ} \mathrm{C}$, $40^{\circ} \mathrm{C}, 60^{\circ} \mathrm{C}, 80^{\circ} \mathrm{C}$ and $100^{\circ} \mathrm{C}$ in a water bath for $2 \mathrm{~h}$ and the temperature stability was scanned (200 nm $-800 \mathrm{~nm})$ in a UV-visible spectrophotometer. The $\mathrm{pH}$ of melanin was adjusted to 4, 6,8,10 using $1 \mathrm{~N} \mathrm{HCl}$ and $1 \mathrm{~N} \mathrm{NaOH}$ let stand for $30 \mathrm{~min}$ at $25^{\circ} \mathrm{C}$. This was scanned for its absorbance using UV-VIS spectrophotometer.

\section{UV-Visible spectra of melanin}

An amount of $0.1 \mathrm{~g}$ of crude melanin was dissolved in $2 \mathrm{~mL}$ of alkaline distilled water (prepared by adding $0.1 \mathrm{~N}$ sodium hydroxide to distilled water) with $\mathrm{pH} 10$. The resulted solution was scanned with UV-VIS spectrophotometer at wavelengths $200-800 \mathrm{~nm}$ and absorption spectra were analysed.

\section{TLC analysis}

The extracted pigment was spotted on TLC and was run in the solvent system consisting of n-butanol, acetic 
acid and water (70:20:10) and the retention factor for pigment by both isolates were measured.

\section{Fourier transform infrared (FTIR)}

FTIR is a rapid and non-destructive technique used for the structural characterization of unknown samples. It is one of the most standout technique used for the detection of functional groups in a compound. The FT-IR spectra of the sample were recorded on FT-IR instrument (ATR-FTIR, Schimadzu Affnity1) in order to characterize the presence of functional groups in isolated pigments. The $2 \mathrm{mg}$ of the isolated compound was added in $200 \mathrm{mg}$ of potassium bromide ( $\mathrm{KBr}-$ FT-IR grade) and prepared as discs pellet. All measurements were carried out in the range of $400-4000 \mathrm{~cm}^{-1}$ at a resolution of $4.0 \mathrm{~cm}^{-1}$.

\section{Antibacterial activity of melanin}

The isolated melanin was subjected to study the antimicrobial activity against 7 human clinical isolates, Staphylococcus aureus, Escherichia coli, Salmonella typhi, Psendomonas aeruginosa, Vibrio parahaemolyticus, Bacillus subtilis, Proteus sp. and Klebsiella sp, isolated from patient samples at a local hospital, Vellore, Tamil Nadu and two foodborne pathogens Listeria monocytogenes (MTCC 657) and Bacillus cereus (MTCC 1272) by 96 well microtiter plate technique. The bacterial loads of overnight cultures were maintained at $1.5 \times 10^{8}$ $\mathrm{CFU} / \mathrm{mL}$ as per the McFarland standards. $50 \mu \mathrm{L}$ of various concentrations of melanin $(10,20,40,60,80,100$ and $150 \mu \mathrm{g} / \mathrm{mL}$ ) was mixed with $50 \mu \mathrm{l}$ of bacterial culture. The plate was incubated for $24 \mathrm{~h}$ at $37^{\circ} \mathrm{C}$ and read at $600 \mathrm{~nm}$.

\section{Anti-biofilm activity of melanin}

The effects of melanin against biofilm forming clinical isolate Staphylococcus sp. and Pseudomonas aeruginosa were tested on 96-well microtiter plate. The melanin at different concentrations $(10,20,40,60,80,100$ and $150 \mu \mathrm{g} / \mathrm{mL}$ ) was added to the well containing bacterial suspensions. The plates were incubated for $24 \mathrm{~h}$ at $37^{\circ} \mathrm{C}$. The incubated plates were washed with sterile phosphate buffer saline (PBS) to remove planktonic cells. This was stained with $0.4 \%$ crystal violet. After staining, the cells were de-stained with $95 \%$ ethyl alcohol. ${ }^{20}$ Bacterial suspensions without melanin were kept as the control system. The contents of the well were quantified spectrophotometrically at $630 \mathrm{~nm}$.

The percentage of biofilm inhibition was calculated by the formula
Percentage inhibition $=\left(A_{C}-A_{t}\right) / A c * 100$

Where $A_{c}$ is the absorbance of control and $A_{t}$ is the absorbance of the test

\section{Anti-quorum sensing activity of melanin}

Anti-quorum sensing activity of melanin was determined by the agar well diffusion method. The agar plates were swabbed with quorum sensing bacterium Chromobacterium violaceum (MTCC 2656). Wells were punched on the agar plates using sterile gel borer. $100 \mu \mathrm{l}$ of various concentrations of melanin $(10,20,40$, $60,80,100$ and $150 \mu \mathrm{g} / \mathrm{mL}$ ) were added to the wells. After 24 to $48 \mathrm{~h}$ of incubation at $37^{\circ} \mathrm{C}$, the zone of inhibition was measured. Kerekes et al. reports the use of agar well diffusion technique to detect the anti-quorum sensing potential of essential oils that possess antibiofilm activity. ${ }^{21}$

\section{Polyphasic identification of the actinobacterial isolate}

The isolate was identified using polyphasic identification including, morphological, biochemical and genomic characterization. Spore chain morphology of both isolates was studied by transplantation embedding technique. The technique was employed to protect the spore chain structure from breaking. The structures were observed under a binocular microscope and as well as scanning electron microscope (SEM- Zeiss [EVO18]). The morphology of the spore surface was also studied to determine the shape and texture of the spore. The samples were sputter coated using $60 \%$ of gold and $40 \%$ of palladium before SEM analysis. The aerial mycelium, substrate mycelium, reverse side pigmentation and soluble pigmentation were studied. The production of melanin and other melanoid pigments were noted. Biochemical tests were performed which included catalase, urease, hydrogen sulfide production, nitrate reduction, gelatin liquefaction, starch hydrolysis tests and IMVIC.

\section{Genomic analysis}

The melanogenic actinobacterial isolates were identified by I6s rRNA sequencing. The isolate was cultured on Yeast Extract Malt Extract Dextrose broth. The genomic DNA was derived using the InstaGene ${ }^{\text {TM }}$ Matrix Genomic DNA isolation kit. Isolated DNA was amplified using MJ Research Peltier Thermo cycler employing universal primers 27F (Forward primer: 5'-AGAGT'TTGATCMTGGCTCAG-3') and 1492R (Reverse primer: 5'-TACGGYTACCT'TGTTACGACTT-3'). The 16srRNA sequences were BLAST (Basic Local Alignment Search Tool) using NCBI BLAST search tool. 


\section{Statistical analysis}

All the experiments performed were replicated in three $(n=3)$ and the results were significant statistically by TWO way ANOVA using GraphPad Prism 6, GraphPad Software, Inc.

\section{RESULTS AND DISCUSSION}

Rare actinobacteria are acclaimed for the production of commercially important secondary metabolites. Researchers are looking forward to more novel metabolites from these microorganisms. Actinobacteria have a significant role in the area of future biotechnology, because of their significance as the producers of bioactive compounds. ${ }^{22}$ Melanin is one such secondary metabolites that microorganisms produce, which they utilize to withstand stressful condition. They are negatively charged, hydrophobic, high molecular weight compounds with amorphous nature. ${ }^{23}$ Melanin production is a widely observed property among lower to higher animals. As compared to the terrestrial actinobacteria, exploration of melanin production by marine actinobacteria is inadequate with limited literature.

Melanin pigment-producing rare actinobacteria were isolated from two different marine soil samples herein. Marine actinomycetes were isolated on Actinomycetes isolation agar, Starch casein agar, ISP7, M1 and M6 media by serial dilution method, heat treatment method and enrichment method. SCA had a significant number of isolates and was regarded as suitable media for the isolation and heat treatment as the suitable technique. $69 \%$ of colonies were observed on SCA. Lee et al. reported similarly the colonies on SCA outnumbered AIA and ISP7. ${ }^{24}$ The isolated actinobacterial colonies were screened for melanin production. Two potent isolates designated as JN1 and JN2 showed dark diffusible pigment in ISP6 and ISP7 media (Figure 1).

Melanin production was also observed in SS broth inoculated with JN1 and JN2 isolates and the pigments were designated as JN1M and JN2M melanin. Melanin production by JN1 and JN2 were initiated at $72 \mathrm{~h}$ and 48 $\mathrm{h}$ of incubation respectively and production increased with the incubation period. After 14 days of incubation, JN2M showed a higher amount of production than JN1M in both static and stationary conditions (Figure 1). The production was correlated with the biomass yield and time of incubation. Whereas, in a study report of Dholakiya et al. a marine strain of Streptomyces cavourensis strain $\mathrm{RD} 8$ was reported to be initiated after $24 \mathrm{~h}$ of growth and reached maximum on the $7^{\text {th }}$ day. ${ }^{25}$ Melanin formation was estimated by dopachrome formation by spectrophotometric means. Dopachrome is a major intermediate in the melanogenic pathway of microorganisms. Therefore, the measurement of dopachrome indirectly measures the production of melanin. The reaction mixtures $(2 \mathrm{~mL}$ of cell-free supernatant + $1 \mathrm{~mL}$ of $0.4 \% \mathrm{~L}-\mathrm{DOPA}$ ) showed black colour after $2 \mathrm{~h}$ of incubation at $37^{\circ} \mathrm{C}$. The absorbance was recorded at $480 \mathrm{~nm}$. JN1 supernatant recorded an absorbance of 0.965 at $480 \mathrm{~nm}$ whereas JN2 supernatant recorded 1.077 which was considered relative in comparison with the previous literature.

Mass production of JN1M was carried out in SS broth under aerobic and shaking condition at room temperature. The optimum carbon and nitrogen sources for JN1 was found be sucrose and tyrosine respectively whereas, JN2 showed optimum production in the media supplemented with lactose and casein (Figure 2). JN1 and JN2 showed variations in melanin production at different temperatures. JN1 and JN2 showed maximum production at $40^{\circ} \mathrm{C}$ and $35^{\circ} \mathrm{C}$ respectively (Figure 3). JN1 showed good melanin production at $\mathrm{pH} 6.0$ and JN2 at neutral pH (Figure 4) Also, melanin production in JN1 and JN2 were found to be higher at $75 \%$ and $100 \%$ of seawater concentration respectively.
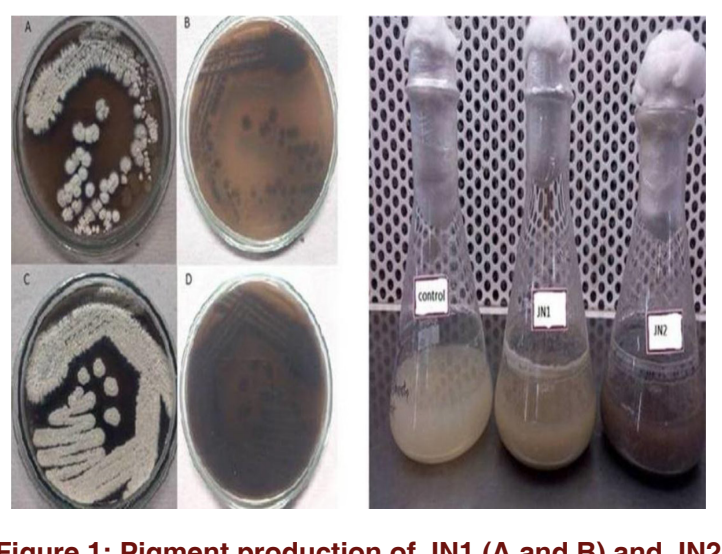

Figure 1: Pigment production of JN1 (A and B) and JN2 (C and D) on ISP7 agar plates; Melanin in SS broth.

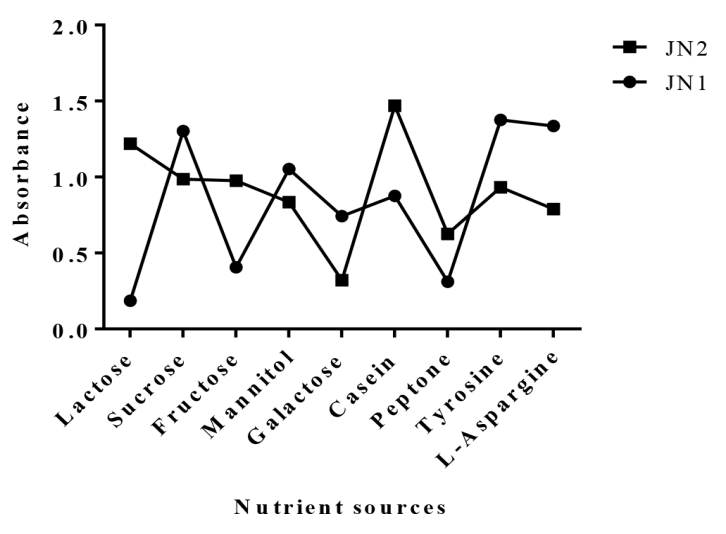

Figure 2: Effect of sugar and nitrogen sources on melanin production. 


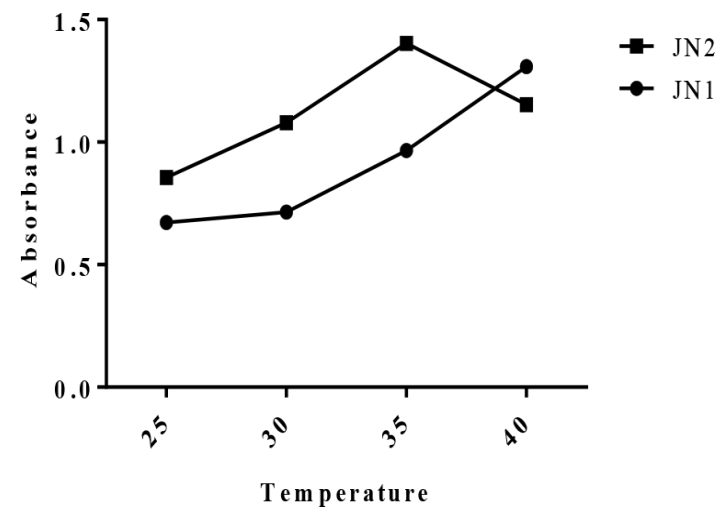

Figure 3: Effect of pH melanin production.

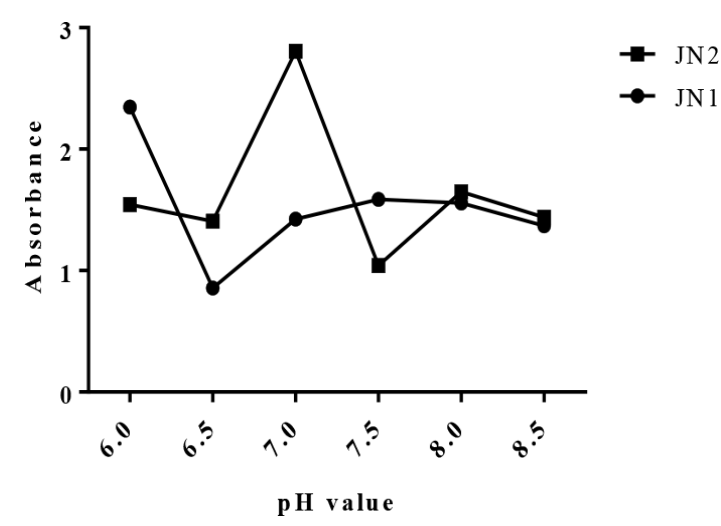

Figure 4: Effect of temperature on melanin production.

The melanin pigment from the isolates JN1 and JN2 were extracted and the dried powder form was used for further analysis. The extracted pigment was determined as melanin by dopachrome formation test and UV-VIS spectrophotometer which showed a peak at $350 \mathrm{~nm}$, whereas a similar study reported the Nocardiopsis melanin peak at $220 \mathrm{~nm}$. Both JN1 and JN2 melanins were found to be stable at a temperature between $20^{\circ} \mathrm{C}$ and $100^{\circ} \mathrm{C}$ and also stable at $\mathrm{pH} 9,10$ and 12. On TLC plates, $\mathrm{R} f$ values of JN1 and JN2 melanins were measured as 0.73 and 0.74 related to melanin pigment as correlated with previous studies. In the study of Kiran et al. they reported the $R f$ value of the purple spots to be $0.74 .{ }^{26}$

We studied the physical properties of the produced pigments. JN1M and JN2M were found to be insoluble in ethyl acetate, chloroform and methanol but they were soluble in dimethylsulfoxide, alkaline water $(\mathrm{pH} 10)$ and phosphate buffer saline $(\mathrm{pH}$ 7.2). The temperature tolerance and stability of JN1M and JN2M were tested and both were found to be stable between temperatures of $20^{\circ} \mathrm{C}$ and $100^{\circ} \mathrm{C}$. The temperature stability was marked by the presence of visible peaks in UV-Spectrophotometer at $350 \mathrm{~nm}$ from 20 to $100^{\circ} \mathrm{C}$.
The investigation of the UV spectrum revealed the absorbance at $350 \mathrm{~nm}$ for both JN1M and JN2M and a diminishing spectrum towards the visible region. The FTIR spectrum of JN1M (Figure 5) was characterized by a broad absorption band at $3259 \mathrm{~cm}^{-1}$ which revealed the presence of alkynes with C-H stretching. The peak at $2929 \mathrm{~cm}^{-1}$ represents $\mathrm{C}-\mathrm{H}$ stretch showing the presence of alkanes and alkyls or O-H stretch showing the presence of carboxylic acids. The peak at $1624 \mathrm{~cm}^{-1}$ was assigned as $\mathrm{N}-\mathrm{H}$ bend related to amines. The peak at $1145 \mathrm{~cm}^{-1}$ shows C-O stretch showing the presence of alcohols. The peak at 1072 has a C-F stretch which falls under alkyl halides. This was correlated with the standard melanin FTIR spectrum and found to be at par.

The FTIR spectrum of JN2M also characterized a broad absorption band at $3240 \mathrm{~cm}^{-1}$ which revealed the presence of $\mathrm{OH}$ stretching and a hydroxyl group. The peak at $2931 \mathrm{~cm}^{-1}$ exhibits a C-H stretch and corresponds to alkanes. The peak at 1629 represents a $\mathrm{C}=\mathrm{O}$ and hence is an aldehyde. The peak at 1409 represents a $\mathrm{C}=\mathrm{C}$ stretch and thus falls in aromatic rings. The peak at $1016 \mathrm{~cm}^{-1}$ and $1145 \mathrm{~cm}^{-1}$ was assigned to $\mathrm{C}-\mathrm{N}$ stretch bond related to the aliphatic amine. This was correlated with the standard melanin FTIR spectrum and found to be at par.

Melanin pigments extracted from microorganisms like bacteria and fungi were reported for their anti-biofilm activity. In the present study, the melanin pigments JN1M and JN2M promising results in the antibiofilm assay with the highest activities of $64.20 \%$ and $65.99 \%$ respectively against Staphylococcus sp. They also showed antibacterial activities against human pathogens with the highest activity of $61.28 \pm 0.280 \%$ and $68.08 \pm 0.141 \%$ against Listeria monocytogenes and Bacillus sp respectively at $150 \mu \mathrm{g} / \mathrm{mL}$, exerted by JN2 (Figure 6). In a similar study, the melanin pigment from Streptomyces was reported to have antibacterial activity against Escherichia coli and Lactobacillus vulgaris. ${ }^{27}$ Our study

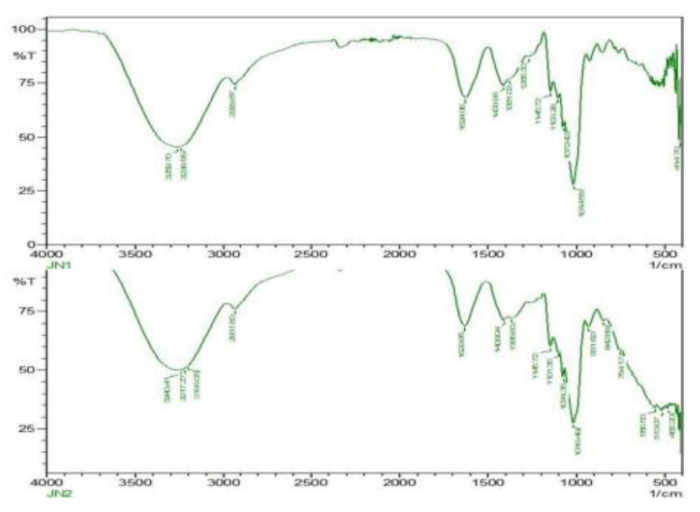

Figure 5: FTIR spectrum of JN1 and JN2 melanin. 


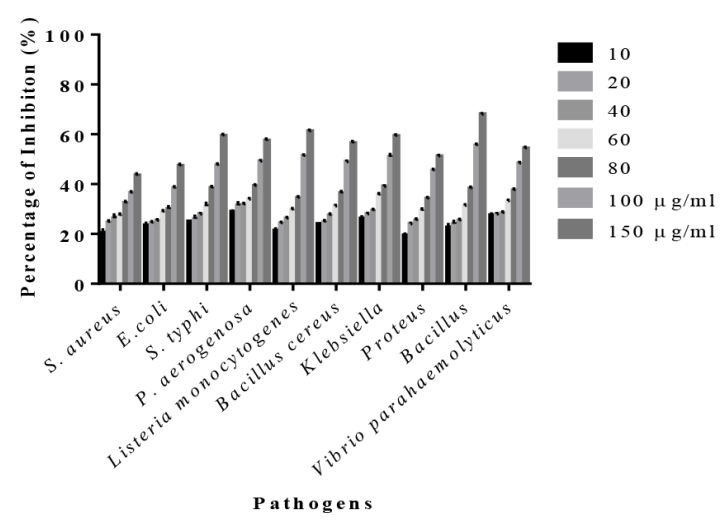

Figure 6: Antimicrobial activity of JN2 melanin.

is the first report regarding the anti-quorum sensing ability of melanin pigment. Both the pigments have inhibited the growth of quorum sensing bacteria Chromobacterium violaceum MTCC 2656 with maximum zones of inhibition of $13 \mathrm{~mm}$ by JN1M and $8 \mathrm{~mm}$ by JN2M at the highest concentration.

The occurrence of melanin production by rare actinobacteria is much lesser when compared to Streptomyces spp. Another study reports the incidence of production of melanin from rare actinobacteria, Actinoalloteichus sp. MHA $15 .{ }^{28}$ However, our study resulted in the isolation of two melanogenic Nocardiopsis species. Nocardiopsis genus is attributed to the production of a wide range of bioactive metabolites exhibiting cytotoxic, antibacterial and antifungal drugs. Ibrahim et al. suggests the percentage of natural products is found as high as $43.55 \%$ in Nocardiopsis spp from marine sediments, followed by terrestrial soil. ${ }^{29}$ The isolates were identified by polyphasic mode of identification. Both the isolates exhibited aerial hyphae in white series. Powdery aerial hyphae were observed for both the isolates grown on ISP7 medium. The substrate hyphae were observed in black colour. JN1 and JN2 were observed as rectus with LPCB staining under optical microscopy. SEM images showed the presence of smooth spores of JN1 and JN2 strains under different magnifications (Figure 7). The colony morphology on the plate, as well as the spore chain morphology, as compared to previous reports resembled Nocardiopsis $s .{ }^{13}$ On performing the biochemical tests, the strains were citrate utilization test positive, catalase test positive. Also, both JN1 and JN2 were found to produce hydrogen sulphide and urease enzyme. Both strains have the ability to reduce the nitrate. However, the two strains were unable to liquefy gelatin. Indole test was negative for both isolates, while Methyl red and Voges Proskauer tests were positive

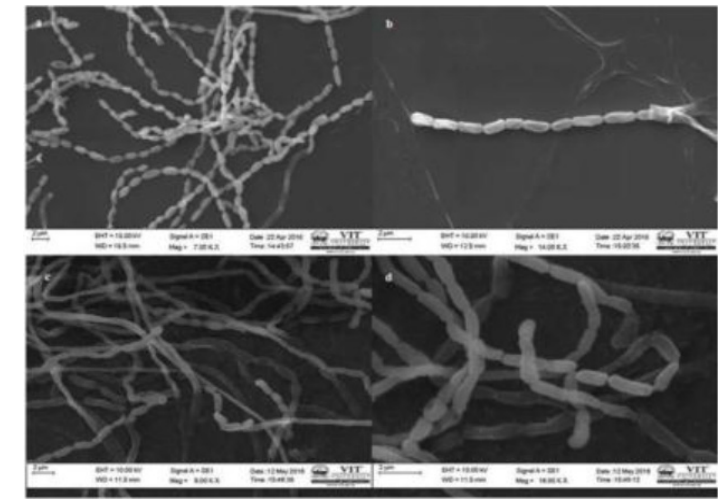

Figure 7: $a$ and $b$ SEM images of JN1 in different magnifications of $7.00 \mathrm{KX}$ and $14.00 \mathrm{KX}$; $\mathrm{C}$ and $\mathrm{d}$ SEM images of JN2 in different magnifications of $9 \mathrm{KX}$ and $18 \mathrm{KX}$.

for JN1 and negative for JN2. Using 16S rRNA sequencing the isolates were identified as Nocardiopsis dassonvillei and Nocardiopsis sp. Furthermore, there are no records stating the melanin production from Nocardiopsis dassonvillei, albeit previous studies had reported the production of melanin from Streptomyces and Nocardiopsis alba. ${ }^{30}$ The potent strains JN1 and JN2 were identified as Nocardiopsis dassonvillei (accession number: KX263302) and Nocardiopsis sp. (accession number: KX263303) respectively by 16s rRNA sequencing (Figure 8).

\section{CONCLUSION}

Melanin is an increasingly important secondary metabolite in pharmaceutical and cosmetic industries. Melanin produced from the isolates herein was compared with previous reports and was concluded as the natural biopigments, melanin. The study opens up the scope of exploring for melanin from Nocardiopsis dassonvillei. The results also depict the prospects of using melanin as a potential anti-quorum sensing agent as there was a significant reduction in the proliferation of the indicator organism, Chromobacterim violaceum MTCC 2656. Thus the exploration of marine habitat has afresh proved to produce potential actinobacteria and also their novel applications.

\section{ACKNOWLEDGEMENT}

The authors thank the management and staff of VIT Vellore, for their technical support in carrying out this research. The authors also thank DST-FIST, India for the SEM facility.

\section{CONFLICT OF INTEREST}

The authors declare no conflict of interest 


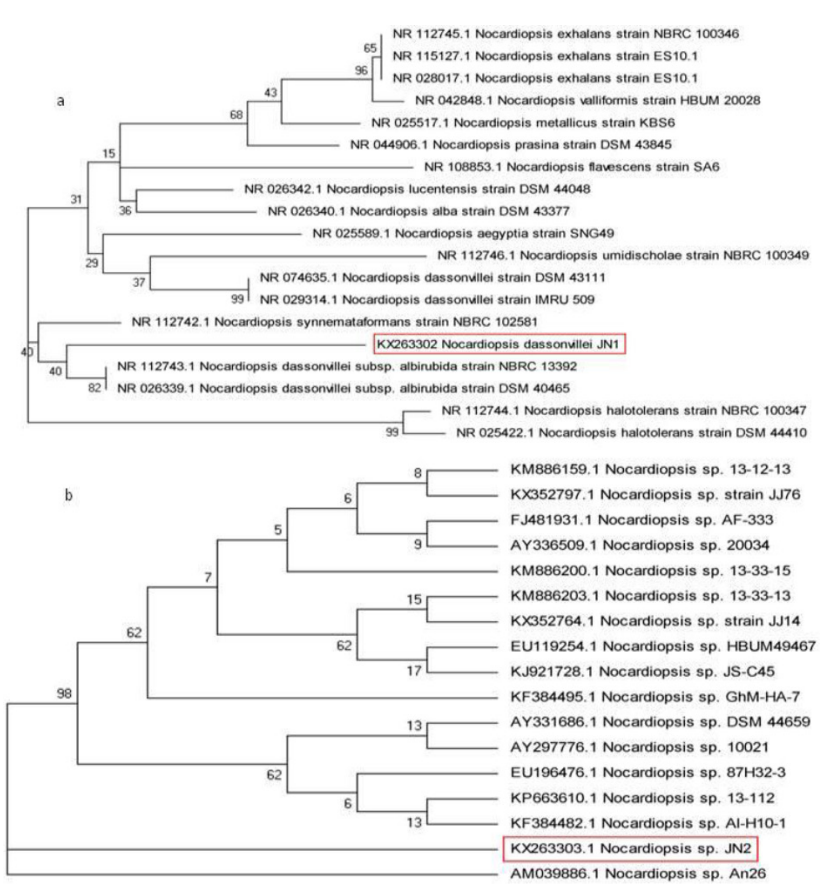

Figure 8: 16s rRNA gene-based phylogenetic tree of strain JN1 (a) and JN2 (b) by neighbour joining method.

\section{ABBREVIATIONS}

ISP6: International Streptomyces Project media no. 6; MTCC: Microbial Type Culture Collection and Gene Bank; nm: Nanometer; rpm: Revolutions per minute; ANOVA: Analysis of Variance.

\section{REFERENCES}

1. Magarvey NA, Keller JM, Bernan V, Dworkin M, Sherman DH. Isolation and characterization of novel marine-derived actinomycete taxa rich in bioactive metabolites. Appl Environ Microbiol 2004;70(2):7520-9.

2. Takizawa M, Colwell RR, Hill RT. Isolation and diversity of actinomycetes in the Chesapeake Bay. Appl Environ Microbiol. 1993;59(4):997-02.

3. Lam KS. Discovery of novel metabolites from marine actinomycetes. Curr Opin Microbiol 2006;9(3):245-51.

4. Berdy J. Bioactive microbial metabolites. J Antibiot. 2003;58(1):1-26.

5. Jensen PR, Williams PG, Oh DC, Zeigler L, Fenical W. Species-specific secondary metabolite production in marine actinomycetes of the genus Salinispora. Appl Environ Microbiol. 2007;73(4):1146-52.

6. Kumar A, Vishwakarma HS, Singh J, Dwivedi S, Kumar M. Microbial pigments: production and their applications in various industries. Int $\mathrm{J}$ Pharm Biol Sci. 2015;5(1):203-12.

7. Joshi VK, Attri D, Baja A, Bhushan S. Microbial pigments. Indian J Biotechnol. 2003;2:362-9.

8. Dastager SG, Li WL, Dayanand A, Tang ST, Tian XP, Zhi XY, et al. Separation, identification and analysis of pigment (melanin) production in Streptomyces. Afr J Biotechnol. 2006;5(11):1131-4.

9. Lerner AB, Fitzpatrick TB. Biochemistry of melanin formation. Physiol Rev. 1950;30(1):91-126.

10. Quadri SR, Agsar D. Detection of melanin-producing thermo-alkaliphilic Streptomyces from limestone quarries of the Deccan traps. World J Sci Technol. 2012;2(2):8-12.

11. Dharmik PG, Gomashe AV. Isolation, identification and antioxidant activity of melanin pigment from actinomycete (Streptomyces species) isolated from garden soil, Nagpur district, India. Int J Pure Appl Sci Technol. 2013;18(1):69-72.

12. Berliner DL, Erwin RL, McGee DR, inventors. Methods of treating Parkinson's disease using melanin. United States Patent US 5210076. 1993.
13. Al-Zarban SS, Abbas I, Al-Musallam AA, Steiner U, Stackebrandt E, Kroppenstedt RM. Nocardiopsis halotolerans sp. nov., isolated from salt marsh soil in Kuwait. Int J Syst Evol Microbiol. 2002;52(2):525-9.

14. Wu Z, Xie L, Xia G, Zhang J, Nie Y, Hu J, et al. A new tetrodotoxin-producing actinomycete, Nocardiopsis dassonvillei, isolated from the ovaries of puffer fish Fugu rubripes. Toxicon 2005;45(7):851-9.

15. Selvin J, Shanmughapriya S, Gandhimathi R, Seghal KG, Rajeetha RT, Natarajaseenivasan $\mathrm{K}$, et al. Optimization and production of novel antimicrobial agents from sponge associated marine actinomycetes Nocardiopsis dassonvillei MAD08. Appl Microbiol Biotechnol. 2009;83(3):435-45.

16. Fu P, Liu P, Qu H, Wang Y, Chen D, Wang $\mathrm{H}$, et al. a-pyrones and diketopiperazine derivatives from the marine-derived actinomycete Nocardiopsis dassonvillei HR10-5. J Nat Prod. 2011;74(10):2219-23.

17. Zhang Q, Li S, Chen Y, Tian X, Zhang H, Zhang G, et al. New diketopiperazine derivatives from a deep-sea-derived Nocardiopsis alba SCSIO 03039. J Antibiot. 2013;66(1):31-6.

18. Arai T, Mikami Y. Chromogenicity of Streptomyces. Appl Environ Microbiol. 1972;23(2):402-6.

19. El-Naggar N, El-Ewasy SM. Bioproduction, characterization, anticancer and antioxidant activities of extracellular melanin pigment produced by newly isolated microbial cell factories Streptomyces glaucescens NEAE-H. Sci Rep. 2017;7:1-19.

20. Bakkiyaraj D, Pandian KST. In vitro and in vivo antibiofilm activity of a coralassociated actinomycete against drug-resistant Staphylococcus aureus biofilms. Biofouling. 2010;26(6):711-7.

21. Kerekes EB, Deak E, Tako M, Tserennadmid R, Petkovits T, Vagv C, et al. Anti-biofilm forming and anti-quorum sensing activity of selected essential oils and their main components on food-related micro-organisms. J Appl Microbiol. 2013;115(4):933-42.

22. Goodfellow M, Williams ST. Ecology of actinomycetes. Annu Rev Microbiol. 1983;37(1):189-216

23. Tarangini K, Mishra S. Production, characterization and analysis of melanin from isolated marine Pseudomonas sp. using vegetable waste. Res J Eng Sci. 2013;2:40-46.

24. Lee L, Zainal N, Azman AS, Eng S, Goh B, Yin W, et al. Diversity and Antimicrobial Activities of Actinobacteria Isolated from Tropical Mangrove Sediments in Malaysia. The Sci World J. 2014;1-14.

25. Dholakiya RN, Kumar MA, Mody KH. Production and Characterization of Melanin from Streptomyces Cavourensis Strain RD8 Using Response Surface Optimization.

26. Kiran GS, Dhasayan A, Lipton AN, Selvin J, Arasu MV, Al-Dhabi NA. Melanintemplated rapid synthesis of silver nanostructures. J Nanobiotechnol. 2014;12(1);1-13.

27. Vasanthabharathi, Lakshminarayanan R, Jayalakshmi S. Melanin production from marine Streptomyces. African J Biotechnol. 2011;10(14):11224-34.

28. Gobalakrishnana R, Sivakumar K. Systematic characterization of potential cellulolytic marine actinobacteria Actinoalloteichus sp. MHA15. Biotechnol Rep. 2017;13:30-6.

29. Ibrahim AH, Desoukey SY, Fouad MA, Kamel MS, Gulder TAM, Abdelmohsen UR. Natural Product Potential of the Genus Nocardiopsis. Mar Drugs. 2018;16(5):147.

30. Sivaperumal P, Kamala K, Rajaram R, Mishra SS. Melanin from marine Streptomyces sp.(MVCS13) with potential effect against ornamental fish pathogens of Carassius auratus. Biocatal Agric Biotechnol. 2014;3(40):134-41. 


\section{Summary}

- Pigments are one of the major bioactive products of actinomycetes, exploited for industrial purpose. Streptomyces species have been increasingly relied on for the extraction of pigments. However, in our study, we concentrate on the extraction of bioactive pigment from rare actinomycetes.

- Two isolates of a rare actinomycetes species, Nocardiopsis dassonvillei and Nocardiopsis sp were isolated and screened to obtain melanin pigment namely JN1M and JN2M respectively.

- Analysis by various techniques viz., thin layer chromatography, UV-Visible spectrophotometry and Fourier Transfer Infra Red spectroscopy confirmed the presence of melanin.

- The assays of bioactivity of the pigment threw light on the anti-biofilm activity. JN1M was found to be inhibiting biofilm forming clinical isolate Staphylococcus sp. showing an activity of $64.20 \pm 3.33 \%$ and pigment from JN2M 65.99 $\pm 2.81 \%$.

- Although anti-bacterial activity was detected, JN2M showed significant antibacterial activities against human pathogens with the highest activity of $61.28 \pm 0.280 \%$ and $68.08 \pm 0.141 \%$ against Listeria monocytogenes and Bacillus sp respectively at $150 \mu \mathrm{g} / \mathrm{mL}$. Anti-quorum sensing ability was further unveiled.

- The study thus establishes the abundance of melanin in rare actinomycetes, making Nocardiopsis sps a source of bioactive melanin.

- The study for the first time reports the anti-quorum sensing activity of melanin.

Cite this article: Kamarudheen N, Naushad T, Rao BKV. Biosynthesis, Characterization and Antagonistic Applications of Extracellular Melanin Pigment from Marine Nocardiopsis Sps. Indian J of Pharmaceutical Education and Research. 2019;53(2S):s112-s120. 